\title{
Toward a Faithful Bidding of Web Advertisement
}

\author{
Takumi Uchida, Koken Ozaki, and Kenichi Yoshida \\ Graduate School of Business Sciences, University of Tsukuba, Japan \\ \{uchida, koken, yoshida\}@gssm. otsuka.tsukuba.ac.jp
}

\begin{abstract}
Web marketing is a key activity of e-commerce. Due to the proliferation of internet technology, available internet marketing data become huge and complex. Efficient use of such large data maximizes the profit of web marketing. Although there are a variety of studies motivated by these backgrounds, there still remains room for improvement on data usage. In this paper, we have proposed a method to realize faithful bidding of web advertisement. The experimental results show: 1) The use of data by the current operators is unreliable, 2) By using the proposed method, the advertisement value of bidding becomes clear. For example, the method could find a cluster of advertisements that has clear cost-effectiveness over other clusters.
\end{abstract}

Keywords: Internet advertisement, allocation of advertising budget, decision support.

\section{Introduction}

Web marketing is a key activity of e-commerce today. Due to the proliferation of internet technology, available internet marketing data become huge and complex. Efficient use of such large data maximizes the profit of web marketing. Although there exist a variety of studies such as [1],[2],[3] motivated by these backgrounds, actual business scenes still rely on the operators' know-how. There still remains room for improvement on data usage.

For example, Fig.1 shows how operators who are working for an advertising agency make their decision on advertisement. They decide the allocation of advertising budget using the Fig.1. X-axis is the number of past actions by the customers. Here, actions are typically web clicks toward the purchase and the installation of software. The target of advertising agency is the maximization of the actions. Y-axis is the budget (costs) used to advertise web pages for the purchase and the software installation. One another target of advertising agency is the minimization of this cost. Cost effectiveness which is typically calculated by $\mathrm{X} / \mathrm{Y}$ (i.e., actions/costs) is important.

An example of know-how which we interviewed from operators of an advertising agency is: "If the current web advertisement is laid out on the lower right segment, increase the budget since the past advertisement worked well (having height cost efficiency)". This know-how is reasonable if the number of data is 


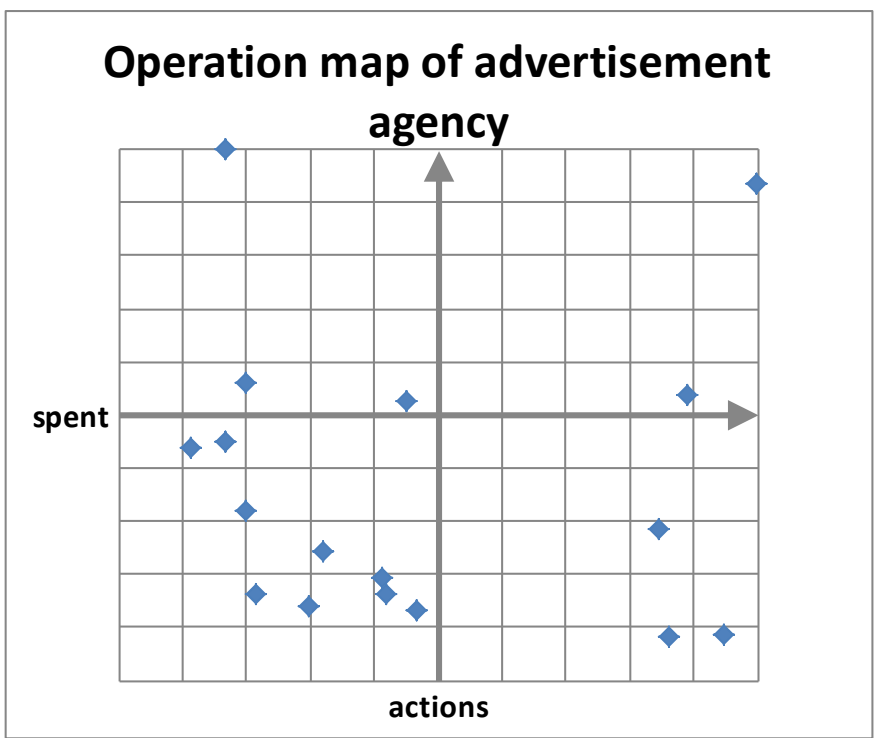

$\mathrm{X}$-axis is number of past actions by the customers such as web clicks toward the purchase and installation of software. $Y$-axis is the budget used to advertise web pages for the actions.

Fig. 1. Operation Map of an Advertisement Agency

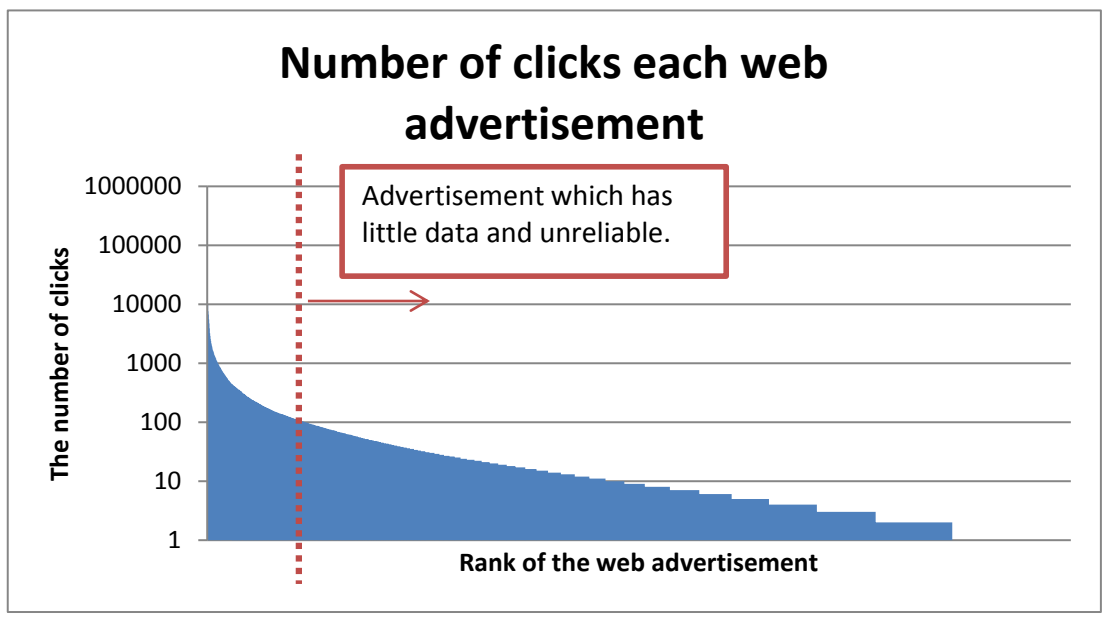

Fig. 2. Number of Clicks for Each Web Advertisements

sufficient and reliable. However, we have found that they don't have enough data in most cases. Fig. 2 shows the fact we found. In Fig.2, Y-axis shows the number 
of clicks for some web advertisement. X-axis shows the rank of the web advertisement in clicks order. Although the total number of data is large, most of the data plotted on Fig.1 has little data and statistically unreliable. The operator use too trifling attributes to plot data on Fig.1. In this study, we propose a method to enlarge the number of data each plot on Fig.1 has. The enlargement increases the statistical reliability of data and increases the adequacy of the operators' judgments.

\section{Evaluating Statistical Reliability of Operators Action}

\subsection{Statistical Background}

Statistical problem of current operator's action is the size of data. Since most of the web advertisement does not have sufficient customer's clicks, the size of data about each web advertisement is small. This makes the operators judgment unreliable. Thus, we develop a method to form groups of similar web advertisements. By merging the data for the similar web advertisements, the number of the data in the resulting cluster becomes large. By using the resulting cluster as the basic unit of decisions, we can realize faithful bidding for each cluster. Following equations give us theoretical background [4]:

$$
\begin{gathered}
\operatorname{Prob}\left(\frac{c}{n}-s \sqrt{\frac{\frac{c}{n}\left(1-\frac{c}{n}\right)}{n}} \leq p \leq \frac{c}{n}+s \sqrt{\frac{\frac{c}{n}\left(1-\frac{c}{n}\right)}{n}}\right) \approx 1-\alpha \\
m=s \sqrt{\frac{\frac{c}{n}\left(1-\frac{c}{n}\right)}{n}} \\
E=\frac{m}{\frac{c}{n}}
\end{gathered}
$$

Here, $\mathrm{c}$ is the number of the observed actions (i.e., purchase or software installation). $\mathrm{n}$ is the number of the observed clicks which users made on the advertisement. $\mathrm{p}$ is the real $\mathrm{n} / \mathrm{c} . \mathrm{s}$ is the approximate value of the each percentile point of the normal distribution. $\mathrm{E}$ is the error of estimated $\mathrm{c} / \mathrm{n}$. To calculate $95 \%$ confidential interval $(\alpha=0.05)$, we set s as 1.96 in this paper. In the rest of this paper, we propose a method which makes clusters of similar advertisement whose E calculated by Eq. (2), i.e., error, is small. By using cluster with small error, we try to realize faithful bidding.

\subsection{Enlarging Cluster}

According to the real data, most of actions/clicks are lower than 5\%. With such data, we try to make a model to predict action from clicks. We assume that the number of actions made by customers follows Poisson distribution [5]. Precisely speaking, we assume following Poisson regression: 


$$
\begin{gathered}
f\left(c_{i}\right)=\frac{\mu_{i}^{c_{i}} e^{-\mu_{i}}}{c_{i} !} \\
\mu_{i}=n_{i} e^{\beta_{0}+\sum_{j=1}^{J} \beta_{j} x_{i j}} \\
\log \mu_{i}=\log n_{i}+\beta_{0}+\sum_{j=1}^{J} \beta_{j} x_{i j}
\end{gathered}
$$

Here, index $\mathrm{i}$ is the cluster-id of advertisements. Each cluster is formed by the advertisements whose attributes share common $x_{i j} . x_{i j}$ is the attributes which specify the characteristics of the advertisement and the users who click that advertisement. Table.1 shows example of attributes. Precisely speaking, since all the attributes we found are categorical attributes, we use binary representation of these attributes. In other words, we actually use attributes $x_{i j}$ each corresponds to the attributes values such as "Tokyo" and "Oosaka". If the value of original attribute "Region" is "Tokyo", the corresponding $x_{i j}$ is set to be 1.

Table 1. Example of Attributes

\begin{tabular}{ll}
\hline Attribute & Value \\
\hline \hline Age & Ex) 10-19, 20-29,,, \\
Region & Ex) Tokyo, Oosaka,,, \\
Sex & Male, Female \\
User Interest & Ex) Fashion, Sports,,, \\
Contents & Ex) Movie, Music,,, \\
\hline
\end{tabular}

$c_{i}$ is the number of the observed actions. $n_{i}$ is the number of the observed clicks which users did on the advertisement. $\mathrm{f}\left(c_{i}\right)$ is probability distribution of actions $c_{i} . \mu_{i}$ is expectation of $c_{i}$ (actions). $\beta$ is regression coefficient. Institutions behind above equations are 1) we can use the number of clicks to estimate the number of customers actions, 2) age, region, and other attributes in Table.1 affect the process of user behavior and affect the conversion process from clicks to actions, 3) Poisson process is reasonable way to represent this process. If the number of actions can be modeled by Poisson process based on the number of clicks and attribute $x_{i j}$, equation (5), i.e., $\mu_{i}$, estimates the number of actions.

Here some of attributes $x_{i j}$ seems to be non-essential. Thus, we try to eliminate non-essential $x_{i j}$ from the equations. We use Akaike's information criterion to eliminate non-essential attributes. We perfume a greedy elimination process. In each step of elimination process, we select attribute $x_{i j}$ which improves AIC index most. This elimination process terminates when none of remaining $x_{i j}$ improves AIC index.

\section{Experimental Results}

To shows the advantage of the proposed method, we have applied the proposed method on the data shown in Fig.2. Fig.3 and 4 show results. Fig.3 shows the 
estimated error for the cluster of advertisements. Here clusters are formed by grouping advertisements with same attributes. All the attributes are used to make clusters for Fig.3. X-axis shows the errors of clusters. It is E calculated by Eq. (3). Y-axis shows the number of actions gained by the advertisement (actions_share). It also shows the total cost for the advertisement (spent_share) and cost-effectiveness (actions_share/spent_share). For example, the height of left most histograms indicates low error rate $(\mathrm{E}<0.2$, i.e. error $<0.2)$. The customer actions won by corresponding advertisements are $62 \%$ with error rate less than 0.2. Although the use of budget on this segment seems to be reasonable, the clusters made with all attributes fail in allocating budget on this segment. Actually, budget used on the same advertisements is only $32 \%$. This result shown in Fig. 3 shows our start point of improvement.

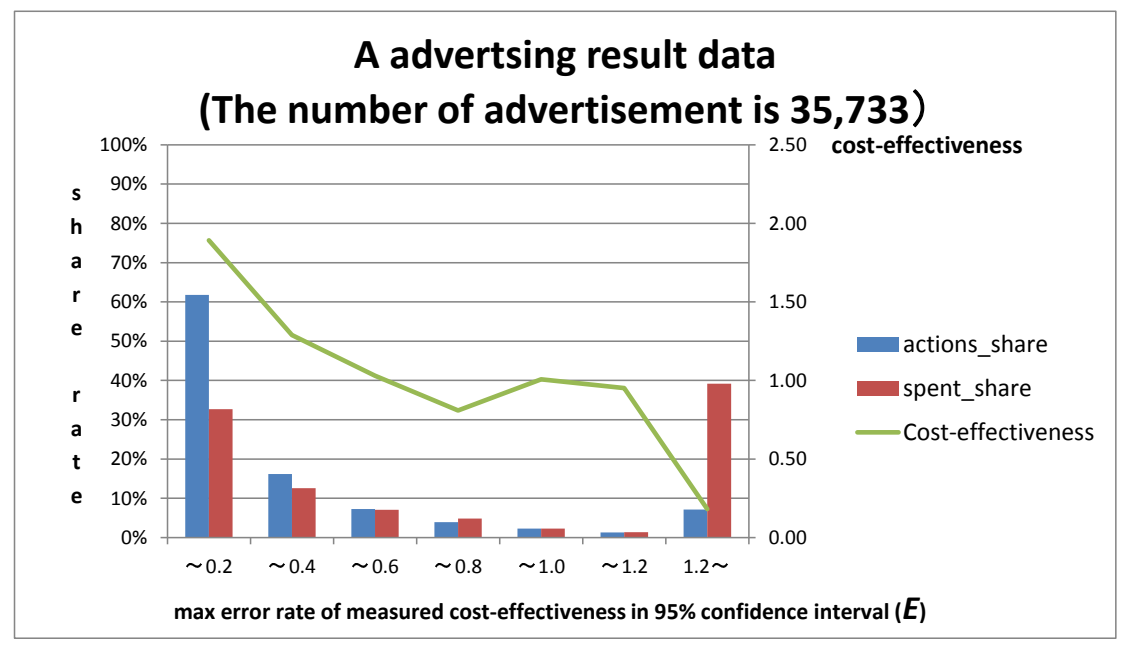

Fig. 3. Reliability of Current Operation

Fig.4 shows the process of improvement by our proposed method. Data shown in Fig. 2 is based on 35,733 advertisements of 177 clients. We have applied the method on data of 177 advertisements of one client company to make Fig.4. The reason we have used the data of only one client is that the value of actions/clicks varies according to the industry. For example, the value of actions/clicks for cosmetics is far larger than that of real estimate. Mixing result of such industries makes the figure unclear. In Fig.4, X-axis is the error of estimated costeffectiveness of operations ( $\mathrm{E}$ of equation 3). $\mathrm{Y}$-axis is the cost-effectiveness (total actions/total cost for the advertisements). Size of the circle is spent_share (total cost of advertisement / total cost of all advertisements). Fig.4 (a) shows the results of clusters formed with all attributes $x_{i j}$ (i.e., start point). Fig.4 (b) shows results of clusters formed with selected attributes $x_{i j}$ (i.e., the results by the proposed method). Fig.4 (c) shows results of clusters formed with randomly 


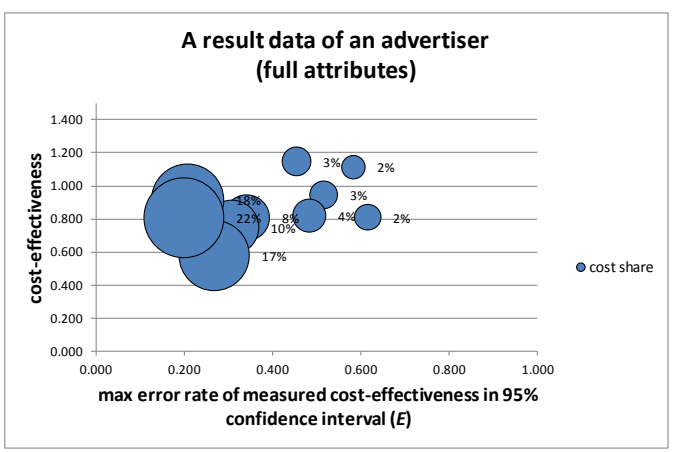

(a) This shows data of an advertiser. Here, No advertisement cluster is reliable. Becaouse, each cluster has little data and unreliable.

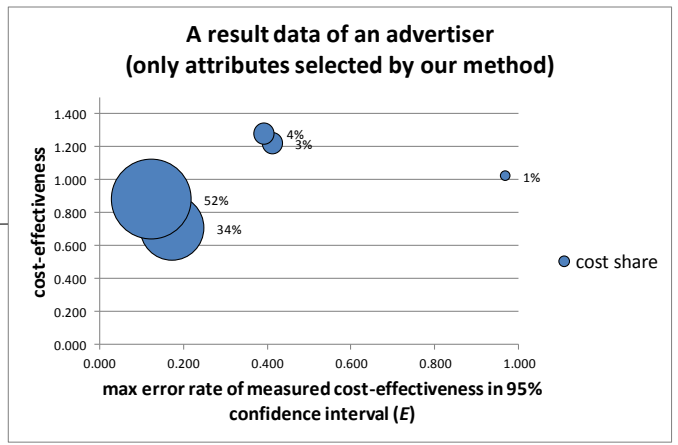

(b) $86 \%$ of cost become reliable. And operators could discover both effective cluster and ineffective cluster which is enough reliable. They could decide on allocatetion.

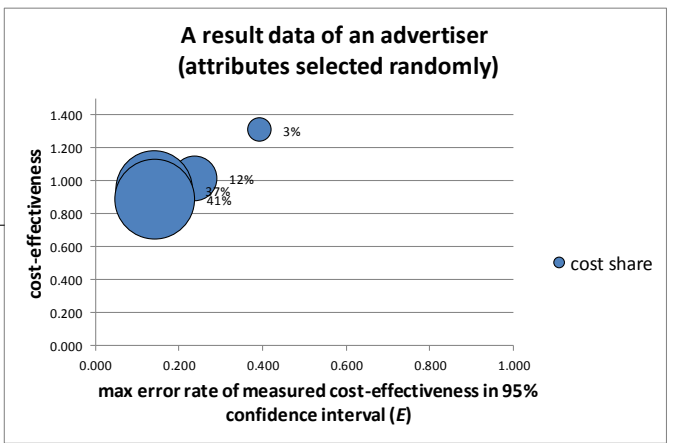

(c) $78 \%$ of cost become reliable. But effectiveness of each cluster which is enough reliable is similar. Thus, they could not decide on allocateic

Fig. 4. Effect of Enlarged Cluster

selected attributes $x_{i j}$ for the comparison purpose. As shown in figures, using all attributes results too many clusters, and all $\mathrm{E}$ of clusters are larger than 0.2 (see Fig.4 (a)). Although, Fig.4 (a) shows the results with slightly larger clusters than that with clusters used in Fig.2, none of cluster has E less than 0.2 . In the 
practical view points, E larger than 0.2 is too large. Thus none of results shown in Fig. 2 has enough accuracy. On the contrary, $86 \%$ of results in Fig.4 (b) have E less than 0.2. This shows the clear improvement of accuracy. Moreover, it shows the fact the one large cluster which has $52 \%$ of advertisements has clear advantage of cost-effectiveness over another large cluster with $34 \%$ of advertisements. Note that this improvement cannot be achieved by random attributes selection (Fig.4 (c)). In Fig.4 (c), $78 \%$ of results have E less than 0.2. However, the found clusters have no clear cost-effectiveness over other clusters. Thus we cannot use the results of Fig.4 (c), i.e., randomly selected attributes.

\section{Conclusion}

In this paper, we have proposed a method to realize faithful bidding of web advertisement. The characteristics of the proposed method are:

- Enlargement of data cluster by removing non-essential attributes during the clustering phase.

- A statistical index is used to select non-essential attributes. Poisson regression analysis and AIC are the theatrical background to select non-essential attributes.

The experimental results show:

- The use of data by the current operators is unreliable. In fact, $67 \%$ of current bidding operations don't have sufficient number of data.

- By using the proposed method, the advertisement value of bidding becomes clear. For example, the method could find a cluster that has clear costeffectiveness over other clusters.

Acknowledgments. This work was partly supported by JSPS KAKENHI Grant Number 25280114.

\section{References}

1. Schlosser, A.E., Shavitt, S., Kanfer, A.: Survey of Internet users' attitudes toward Internet advertising. Journal of Interactive Marketing 13(3), 34-54 (1999)

2. Manchanda, P., Dube, J.-P., Goh, K.Y., Chintagunta, P.K.: The Effect of Banner Advertising on Internet Purchasing. Journal of Marketing Research 43(1), 98-108 (2006)

3. Shabbir, G., Niazi, K., Siddiqui, J., Shah, B.A., Hunjra, A.I.: Effective advertising and its influence on consumer buying behavior. MPRA Paper No. 40689 (August 2012)

4. Hogg, R.V., McKean, J.W., Craig, A.T.: Introduction to Mathematical Statistics, 6th edn. Person Education, Inc. (June 2004)

5. Dobson, A.J.: An Introduction to Generalized Linear Models, ch. 9, 3rd edn. Chapman and Hall/CRC (November 2001) 